\title{
Quantum Monte Carlo study of small pure and mixed spin-polarized tritium clusters
}

\author{
I. Bešlić \\ Faculty of Natural Sciences, University of Split, HR-21000 Split, Croatia \\ L. Vranješ Markić \\ Faculty of Natural Sciences, University of Split, HR-21000 Split, Croatia and \\ Institut für Theoretische Physik, Johannes Kepler Universität, A-4040 Linz, Austria \\ J. Boronat \\ Departament de Física i Enginyeria Nuclear, Campus Nord B4-B5, \\ Universitat Politècnica de Catalunya, E-08034 Barcelona, Spain
}

(Dated: October 30, 2018)

\begin{abstract}
We have investigated the stability limits of small spin-polarized clusters consisting of up to ten spin-polarized tritium $\mathrm{T} \downarrow$ atoms and the mixtures of $\mathrm{T} \downarrow$ with spin-polarized deuterium $\mathrm{D} \downarrow$ and hydrogen $\mathrm{H} \downarrow$ atoms. All of our calculations have been performed using the variational and diffusion Monte Carlo methods. For clusters with $\mathrm{D} \downarrow$ atoms, the released node procedure is used in cases where the wave function has nodes. In addition to the energy, we have also calculated the structure of small clusters using unbiased estimators. Results obtained for pure $\mathrm{T} \downarrow$ clusters are in good accordance with previous calculations, confirming that the trimer is the smallest spinpolarized tritium cluster. Our results show that mixed $\mathrm{T} \downarrow-\mathrm{H} \downarrow$ clusters having up to ten atoms are unstable and that it takes at least three tritium atoms to bind one, two or three $\mathrm{D} \downarrow$ atoms. Among all the considered clusters, we have found no other Borromean states except the ground state of the $\mathrm{T} \downarrow$ trimer.
\end{abstract}

PACS numbers: 67.65.+z, 02.70.Ss, 36.40.-c 


\section{INTRODUCTION}

Electron spin-polarized hydrogen $(\mathrm{H} \downarrow)$ has extreme quantum nature due to its very small mass and weakly attractive potential. It remains in gas phase even in the limit of zero temperature and up to pressures of about 170 bar, $\frac{1}{-}$ after which it solidifies. Stwalley and Nosanow $^{2}$ suggested in 1976 the use of $\mathrm{H} \downarrow$ for achieving a Bose-Einstein condensate (BEC) state, which was finally achieved for hydrogen in 1998 by Fried et al..$\underline{3}$ Heavier isotopes of hydrogen, spin-polarized deuterium $(\mathrm{D} \downarrow)$ and tritium $(\mathrm{T} \downarrow)$ also show a remarkable quantum behavior. $\mathrm{D} \downarrow$ atoms, which obey Fermi statistics, have nuclear spin one and thus the zeropressure state of bulk $\mathrm{D} \downarrow$ depends on the number of occupied nuclear spin states. Previous theoretical estimations $4, \underline{5}, \underline{6}$ have shown that $\left(\mathrm{D} \downarrow_{1}\right)$ with only one occupied nuclear spin state is gas at zero pressure, while bulk $\mathrm{D} \downarrow$ with two $\left(\mathrm{D} \downarrow_{2}\right)$ and three $\left(\mathrm{D} \downarrow_{3}\right)$ equally occupied nuclear spin states remains liquid at zero pressure and zero temperature. Spin-polarized tritium, which obeys Bose statistics, is expected to be liquid ${ }^{7,8}, \underline{9}$ due to its larger mass.

Recently, Blume et al. $\stackrel{10}{\underline{n}}$ have studied microscopic properties of tritium $(\mathrm{T} \downarrow)_{N}$ clusters with up to $N=40$ atoms using the diffusion Monte Carlo (DMC) method and compared them to bosonic ${ }^{4} \mathrm{He}_{N}$ clusters. In that work, it is shown that tritium clusters $(\mathrm{T} \downarrow)_{N}$ are even more weakly bound than helium clusters ${ }^{4} \mathrm{He}_{N}$ with the same number of atoms. Furthermore, their results suggest the use of $\mathrm{T} \downarrow$ as a new BEC gas with the advantage of a nearly exact knowledge of its interatomic potential and the possibility of manipulating the strength of the interactions via Feshbach resonances. In addition, it has been shown $\underline{10}$ that the trimer $(\mathrm{T} \downarrow)_{3}$ is the smallest spin-polarized tritium cluster with a ground-state energy of only $-4.2(7) \mathrm{mK}$. It is thus an example of Borromean or halo state, $, 11,12$ because the two-body T $\downarrow$ system does not have a bound state. The prediction of a total zero angular momentum bound state of the spin-polarized atomic tritium trimer has been recently confirmed by Salci et al. $\stackrel{13}{ }$ using the finite element method. Importantly, the use of this accurate few-body approach leads to conclude that there are not any other bound or shape resonant states with zero and nonzero angular momentum. 13

Unlike the case of pure ${ }^{3} \mathrm{He},{ }^{4} \mathrm{He}$ and mixed ${ }^{3} \mathrm{He}-{ }^{4} \mathrm{He}$ clusters, which have been the subject of both experimental and theoretical study, 14,15,16,17,18 nothing is known about the stability of mixed or other pure spin-polarized hydrogen clusters different from tritium ones. ${ }^{3} \mathrm{He}$ has almost the same mass as $\mathrm{T} \downarrow$, but because of its small mass and Fermi statistics it takes at least $30{ }^{3} \mathrm{He}$ atoms to form a bound state. $\underline{18}$ Recently, Hanna and Blume $\frac{19}{}$ have studied the near-threshold behavior of weekly bound three-dimensional bosonic clusters with up to 40 atoms interacting additively through two-body van der Waals potentials modeled after the $\mathrm{T} \downarrow$ - $\mathrm{T} \downarrow$ interaction. Among other results, they found a four-parameter fit for the critical mass needed to bind a bosonic cluster of $N$ atoms. From this, it follows that if $\mathrm{D} \downarrow$ were a boson, due to its smaller mass compared to $\mathrm{T} \downarrow$, it would take at least 13 atoms to form a bound cluster $(\mathrm{D} \downarrow)_{N}$. Considering that $\mathrm{D} \downarrow$ obeys Fermi statistics we expect that, similarly to the case of ${ }^{3} \mathrm{He},{ }^{18}$ it will take more than $30 \mathrm{D} \downarrow$ atoms to form a self-bound cluster. On the other hand, although $\mathrm{H} \downarrow$ obeys Bose statistics, its approximately three times larger zero-point energy (with respect to $\mathrm{T} \downarrow$ ) implies that clusters of $\mathrm{H} \downarrow$ are not stable. $\frac{19}{}$ This statement is in agreement with the gas nature of bulk $\mathrm{H} \downarrow$ at zero temperature. $\underline{\underline{1}}$ Therefore, hydrogen-tritium and deuterium-tritium clusters are reasonable choices for getting stable self-bound spin-polarized clusters.

If tritium could be experimentally prepared to achieve a BEC state, as suggested in Ref. 10, the presence of a finite fraction of deuterium would be a new example of a Bose- 
Fermi mixture with BEC. In fact, Bose-Fermi mixtures composed with alkalines are nowadays studied in several laboratories and the main physics underlying them is well known. $\underline{20}$ Tritium-deuterium mixtures would be even more interesting since they are isotopic and with a very well known interaction. Dilute vapors are also candidates for searching the elusive Efimov states, ${ }_{21}$ and recently experimental evidence of its existence has been observed in an ultracold gas of cesium atoms. ${ }^{22}$ In the case of pure tritium, it has been proved that such state does not exist 10 but mixed isotopic hydrogen systems could offer new possibilities for continuing the search of trimers with Efimov character.

In this article, we report energetic and structural properties of small spin-polarized tritium-deuterium and tritium-hydrogen clusters, obtained using the diffusion Monte Carlo method. It is our main goal to determine which of these clusters are stable. As many of them extend beyond the classically forbidden region they can be considered as quantum halo systems. We are particularly interested in finding among these halo clusters mixed Borromean or "super-Borromean" 19 systems for which all subsystems are unbound. So far, ${ }^{3} \mathrm{He}_{2} \mathrm{~K}$ is the only mixed molecular system for which the Borromean state has been predicted theoretically. $\underline{\underline{23}}$

In Sec. II, we briefly describe the DMC method and the trial wave functions used for importance sampling. Due to the fermionic nature of $\mathrm{D} \downarrow$, the fixed-node and released-node methods are used in cases where the wave function has nodes. Sec. III reports the results obtained, and finally, Sec. IV comprises the summary of the results and main conclusions.

\section{METHOD}

We consider that all the $\mathrm{H} \downarrow, \mathrm{D} \downarrow$, and $\mathrm{T} \downarrow$ atoms interact with the spin-independent central triplet pair potential $b^{3} \Sigma_{u}^{+}$. This interaction has been determined in an essentially exact way by Kolos and Wolniewicz, ${ }^{24}$ and recently extended to larger interparticle distances by Jamieson et al. (JDW) ${ }^{25}$ We have used a cubic spline interpolation between JDW data and smoothly connected the points to the long-range behavior as calculated by Yan et al.. ${ }^{26}$ The same form has been used in the recent DMC calculation of bulk $\mathrm{H} \downarrow, \underline{1}$, work in which a comparison between different potentials employed in the past is also reported. On the other hand, we have checked that the addition of mass-dependent adiabatic corrections (as calculated by Kolos and Rychlewski 27 ) to the JDW potential does not modify the energy of spin-polarized hydrogen clusters.

The starting point of the DMC method is the Schrödinger equation written in imaginary time,

$$
-\hbar \frac{\partial \Psi(\boldsymbol{R}, t)}{\partial t}=(H-E) \Psi(\boldsymbol{R}, t)
$$

where $E$ is a constant acting as a reference energy, $\boldsymbol{R} \equiv\left(\boldsymbol{r}_{1}, \ldots, \boldsymbol{r}_{N}\right)$ is a walker in Monte Carlo terminology, and $H$ is the $N$-particle Hamiltonian

$$
H=-\sum_{i=1}^{N} \frac{\hbar^{2}}{2 m_{i}} \nabla_{i}^{2}+\sum_{i<j}^{N} V\left(r_{i j}\right)
$$

As usual in the method, a trial wave function $\psi(\boldsymbol{R})$ is introduced for importance sampling and then the Schrödinger equation (1) is rewritten in terms of $\Phi(\boldsymbol{R}, t)=\Psi(\boldsymbol{R}, t) \psi(\boldsymbol{R})$. The resulting equation is solved stochastically by considering a short-time approximation for the 
Green's function. In the limit $t \rightarrow \infty$ (for long simulation times), only the lowest energy eigenfunction not orthogonal to $\psi(\mathbf{R})$ survives and then the sampling of the ground state is effectively achieved.

The trial wave function used for the simulation of the pure $T \downarrow$ clusters is of Jastrow form, $\psi_{\mathrm{J}}(\boldsymbol{R})=\prod_{i<j}^{N} f_{T}\left(r_{i j}\right)$, where $f_{T}\left(r_{i j}\right)$ describes correlations between pairs of tritium atoms. Similarly, wave functions for the study of mixed tritium-hydrogen clusters, containing $N_{1}$ $\mathrm{H} \downarrow$ atoms and $N_{2} \mathrm{~T} \downarrow$ atoms $\left(N_{1}+N_{2}=N\right)$, are constructed as a product of two-body correlation functions between all the pairs,

$$
\psi\left(\mathbf{R} ; N_{1}, N_{2}\right)=\prod_{\substack{i, j=1 \\ i<j}}^{N_{1}} f_{H}\left(r_{i j}\right) \prod_{\substack{i, j=N_{1}+1 \\ i<j}}^{N} f_{T}\left(r_{i j}\right) \prod_{i=1}^{N_{1}} \prod_{j=N_{1}+1}^{N} f_{H T}\left(r_{i j}\right)
$$

where $f_{H}(r)$ describes the two-body correlations between $\mathrm{H} \downarrow$ atoms and $f_{H T}(r)$ accounts for the $\mathrm{H} \downarrow-\mathrm{T} \downarrow$ pairs. In case of mixed $(\mathrm{D} \downarrow)_{N_{1}}(\mathrm{~T} \downarrow)_{N_{2}}$ clusters, the total wave-function is constructed as a product of an antisymmetric function corresponding to deuterium $\left(\psi_{A}\right)$ and Jastrow factors,

$$
\psi\left(\mathbf{R} ; N_{1}, N_{2}\right)=\psi_{A} \prod_{\substack{i, j=1 \\ i<j}}^{N_{1}} f_{D}\left(r_{i j}\right) \prod_{\substack{i, j=N_{1}+1 \\ i<j}}^{N} f_{T}\left(r_{i j}\right) \prod_{i=1}^{N_{1}} \prod_{j=N_{1}+1}^{N} f_{D T}\left(r_{i j}\right)
$$

where $f_{D}(r)$ describes two-body correlations between $\mathrm{D} \downarrow$ atoms and $f_{D T}(r)$ two-body correlations for the $\mathrm{D} \downarrow-\mathrm{T} \downarrow$ pairs. For the function $\psi_{A}$ it is enough to consider the product of Slater determinants for each one of the nuclear spin states. Consequently, $\psi_{A}$ has different form in the case of $\mathrm{D} \downarrow_{2}$ and $\mathrm{D} \downarrow_{3}$ mixed $\mathrm{D} \downarrow$-T $\downarrow$ clusters. For example, in the case of $\left(\mathrm{D} \downarrow_{2}\right)_{N_{1}}(\mathrm{~T} \downarrow)_{N_{2}}$ clusters, $\psi_{A}=1$ for $N_{1}=1,2, \psi_{A}=x_{1}-x_{2}$ for $N_{1}=3$, and $\psi_{A}=\left(\mathbf{r}_{1}-\mathbf{r}_{2}\right)\left(\mathbf{r}_{3}-\mathbf{r}_{4}\right)$ for $N_{1}=4$, while for $\left(\mathrm{D} \downarrow_{3}\right)_{N_{1}}(T \downarrow)_{N_{2}}$ clusters $\psi_{A}=1$ for $N_{1}=1,2,3, \psi_{A}=x_{1}-x_{2}$ for $N_{1}=4$ and $\psi_{A}=\left(\mathbf{r}_{1}-\mathbf{r}_{2}\right)\left(\mathbf{r}_{3}-\mathbf{r}_{4}\right)$ for $N_{1}=5$.

We have worked with three different models for the two-body correlation function $f_{T}(r)$,

$$
\begin{gathered}
f_{T}(r)=\exp \left[-b_{1} \exp \left(-b_{2} r\right)-b_{3} r\right], \\
f_{T}(r)=\frac{1}{r} \exp \left[-\left(\frac{\alpha}{r}\right)^{\gamma}-s r\right], \\
f_{T}(r)=\exp \left[-\left(\frac{b}{r}\right)^{5}-s r\right],
\end{gathered}
$$

where $b_{1}, b_{2}, b_{3}, \alpha, \gamma, s$ and $b$ are variational parameters. They have been obtained by optimizing the variational energy calculated with the VMC method. Finally, we found the form (6) to be optimal for clusters from three to five $T \downarrow$ atoms, while the other two (5.7) suit better for larger clusters. The same function, with different variational parameters, has then been used for all the other two-body correlation functions $\left(f_{H}(r), f_{D}(r), f_{H T}(r), f_{D T}(r)\right)$. For example, in case of pure $\mathrm{T} \downarrow$ clusters $(\alpha, \gamma, s)$ range from $(3.9,4.0,0.0012)$ for trimer to $(4.0,3.7,0.001)$ for $(\mathrm{T} \downarrow)_{5}$ and $(b, s)$ range from $(3.55,0.065)$ for $(\mathrm{T} \downarrow)_{6}$ to $(3.6,0.05)$ for $(\mathrm{T} \downarrow)_{10}$. The VMC recovers from $70 \%$ to $90 \%$ of the DMC energy, except in case of $(\mathrm{T} \downarrow)_{3}$ where the VMC energy is only $-0.85(8) \mathrm{mK}$. In case of mixed clusters the parameters in the $f_{T}(r)$ function do not change significantly, so for example in case of the form (7) $b$ assumes values 
from 3.5 to 3.62 and $s$ from 0.07 to 0.05 , going from smaller to larger clusters, respectively. At the same time, in the function $f_{T H}(r), b$ goes from 3.58 to 3.7 and $s$ is around 0.003 . Similar behavior of variational parameter $b$ is obtained for the other correlation functions, while $s$ is around 0.01 in $f_{D T}(r)$ and around 0.003 in $f_{D}(r)$. In most cases of mixed clusters VMC recovers from $60 \%$ to $90 \%$ of the DMC energy, exceptions being smaller clusters with more than two $\mathrm{D} \downarrow$ atoms and the clusters having only three $\mathrm{T} \downarrow$ atoms, where the VMC energies are further from the DMC ones.

The DMC method we have used is accurate to second order in the time step $\Delta t t^{28}$ The ground-state energies have been calculated for several time-steps and then extrapolated to zero $\Delta t$ to remove any possible time-step bias. We have also studied the optimal mean walker population and finally chosen $N_{w}=1000$ in order to eliminate any bias coming from it.

Apart from statistical uncertainties, the energy of the bosonic clusters is exactly calculated. In the case of mixed clusters with more than three $\mathrm{D} \downarrow_{3}$ or two $\mathrm{D} \downarrow_{2}$ atoms, the sign problem appears because the trial wave function changes sign. Since the Monte Carlo method requires that $f(\mathbf{R}, t)=\Psi(\mathbf{R}, t) \psi(\mathbf{R}) \geq 0$, we have first used the so-called fixed-node approximation which allows only the moves in which $\psi$ and $\Psi$ change sign together, thus fixing the nodes. In this way, an upper bound to the energy is obtained. ${ }^{29}$ In a subsequent step, we have removed the nodal constraint imposed by the trial wave function by using the released-node method. We have adopted the methodology used previously in liquid helium calculations,, 30 and recently employed in the study of small mixed helium clusters $\frac{17}{}$ In this approach, walkers are allowed to cross the nodes imposed by the trial wave function and survive for a finite lifetime. In order to achieve an effective crossing of the nodal surface an auxiliary guiding wave function $\psi_{g}$ is introduced in the DMC calculation. This function is positive everywhere, different from zero in the nodes, and approaches $|\psi|$ away from the nodes. Like in Refs. 17, 30, we have taken $\psi_{g}=\psi_{J}(\mathbf{R})\left(\psi_{\mathbf{A}}^{\mathbf{2}}(\mathbf{R})+a^{\mathbf{2}}\right)^{\mathbf{1} / \mathbf{2}}$, which satisfies the above condition for suitable choices of $a$. The released-node energy is estimated through an exponential fit $E(t)=E_{r}+A e^{-(t / \tau)}$ to the DMC data, with $t$ the released time. In all cases where the RN method is used in this work the difference between the last calculated point in released time and $E_{r}$ is of the same order as the statistical noise. The behavior of $a$ and the maximum released time needed to achieve the asymptotic limit of the released energy has been similar to the case studied in Ref. 17. Also, like in Ref. 17, we find the RN energies to be very close to the FN ones. In addition, in the case of $(T \downarrow)_{4}\left(D \downarrow_{2}\right)_{3}$ we have verified that the inclusion of backflow correlations in $\psi_{A}$ does not change the final FN results.

\section{RESULTS}

DMC results for the ground state energy of pure and mixed $\mathrm{T} \downarrow-\mathrm{H} \downarrow$ clusters are given in Table I. Our results for pure tritium $\mathrm{T} \downarrow$ clusters are compared to the results of Blume et. $a l l^{10}$ in Fig. 1. We obtain a good agreement with these published data, although our results are slightly lower for all $N$. Underlying this difference is the fact that in Ref. 10 a damped three-body Axilrod-Teller potential term ${ }^{31}$ is introduced in the Hamiltonian, raising the trimer energy $1.6 \%$ and the energy of the cluster with 40 particles $6 \%$. We also find that the trimer is the smallest spin-polarized tritium cluster with an energy of only $-4.8(2) \mathrm{mK}$, confirming it to be a halo state. In order to test the sensitivity of the present results to the details in the interaction potential, we have also calculated the energy with the potential that Silvera and Goldman ${ }^{32}$ constructed as a fit to the older Kolos and Wolniewicz 
data. ${ }^{24}$ It is worth mentioning that a full comparison between the different potentials used in the literature for studying bulk $\mathrm{H} \downarrow$ can be found in the Ref. 1. Using the Silvera model, we obtain a trimer binding energy significantly smaller, $-1.9(4) \mathrm{mK}$. The trimer energy is therefore sizably affected by the interatomic potential and the reason for that lies on the huge cancellation between the kinetic and potential energies. For instance, using the JDW potential and the pure estimator for the potential energy, we obtain $E_{p}=-355(9)$ $\mathrm{mK}$ and $E_{k}=350(9) \mathrm{mK}$. For larger clusters, the influence of the potential form on the binding strength is reduced: for the tetramer and with the Silvera potential, we obtain an energy $E=-107(2) \mathrm{mK}$ which is only about $15 \%$ weaker than the one for the JDW potential, $E=-126(2) \mathrm{mK}$. The potential and kinetic energies of the tetramer using the JDW potential are $E_{p}=-1871(11) \mathrm{mK}$ and $E_{k}=1745(12) \mathrm{mK}$.

The addition of one $\mathrm{H} \downarrow$ atom to the $(\mathrm{T} \downarrow)_{N}$ cluster creates a system that appears to be at the threshold of stability or unstable, that is the ground-state energy of the $(\mathrm{T} \downarrow)_{N} \mathrm{H} \downarrow$ cluster is within the errorbars equal to or higher than the energy of the $(T \downarrow)_{N}$ cluster, respectively. In order to be certain about the conclusion concerning the stability of the $(\mathrm{T} \downarrow)_{N} \mathrm{H} \downarrow$ clusters, we have repeated the calculations using two different types of trial wave functions. In the case where VMC energies are further away from the DMC ones, a lot of simulation time is needed for the system to reach the equilibrium state, and in some cases with the trial function (6) the lowering of the energy with imaginary time going on is almost imperceptible. On the other hand, with both (5) and (7), and for small values of the parameter $s$ or $b_{3}$ in the $f\left(r_{T H}\right)$ function, the system rather quickly reaches the equilibrium energy, which is within the errorbars equal to the energy of the system without the $\mathrm{H} \downarrow$ atom. Furthermore, if we continue the simulation after the system has reached the ground-state energy, we can observe the surplus $\mathrm{H} \downarrow$ atom leaving the cluster. This indicates that these clusters are effectively unstable. We have also calculated the ground-state energies of several clusters with more hydrogen atoms $(\mathrm{T} \downarrow)_{N}(\mathrm{H} \downarrow)_{M}$, such that $N+M \leq 10$. Our results indicate that these clusters are also unstable, because although in some cases negative energies are obtained they are above those for $(\mathrm{T} \downarrow)_{N} \mathrm{H} \downarrow$.

Table II presents the results for the ground-state energy of mixed $\mathrm{T} \downarrow$ - $\mathrm{D} \downarrow$ clusters. Due to the approximately twice larger mass of $\mathrm{D} \downarrow$ compared to $\mathrm{H} \downarrow$, clusters with one or two $\mathrm{D} \downarrow$ atoms and at least three $\mathrm{T} \downarrow$ atoms are stable. In the clusters $(\mathrm{T} \downarrow)_{N}(\mathrm{D} \downarrow)_{2}$, we have assumed that $\mathrm{D} \downarrow$ atoms occupy two different nuclear spin states. For clusters $(\mathrm{T} \downarrow)_{N}(\mathrm{D} \downarrow)_{M}$ with $M>2$, we have considered both the case where two different nuclear spin states are occupied $(\mathrm{T} \downarrow)_{N}\left(\mathrm{D} \downarrow_{2}\right)_{M}$ and the case with three different occupied nuclear spin states $(\mathrm{T} \downarrow$ )$_{N}\left(\mathrm{D} \downarrow_{3}\right)_{M}$. Similar to the case of the bulk system,,$+\frac{4,6}{6}$ we find that the three-component spin clusters are more strongly bound than the ones where $\mathrm{D} \downarrow$ occupies two nuclear spin states, $E\left((\mathrm{~T} \downarrow)_{N}\left(\mathrm{D} \downarrow_{3}\right)_{M}\right)<E\left((\mathrm{~T} \downarrow)_{N}\left(\mathrm{D} \downarrow_{2}\right)_{M}\right)$. Furthermore, $(\mathrm{T} \downarrow)_{N}\left(\mathrm{D} \downarrow_{3}\right)_{3}$, whose spatial wave function has no nodes, are all stable for $N \geq 3$, while it takes $6 \mathrm{~T} \downarrow$ atoms to form a stable $(\mathrm{T} \downarrow)_{N}\left(\mathrm{D} \downarrow_{2}\right)_{3}$ cluster. The smaller $(\mathrm{T} \downarrow)_{N}\left(\mathrm{D} \downarrow_{2}\right)_{3}$ clusters have the same energy as the $(\mathrm{T} \downarrow)_{N}(\mathrm{D} \downarrow)_{2}$ clusters within the errorbars, but the analysis of the distributions shows one of the $\mathrm{D} \downarrow$ atoms leaving the system. The clusters $(\mathrm{T} \downarrow)_{N}\left(\mathrm{D} \downarrow_{3}\right)_{4}$ are similarly at the threshold of binding for $N=3,4,5,6$, just like the clusters $(\mathrm{T} \downarrow)_{N}\left(\mathrm{D} \downarrow_{2}\right)_{4}$, and again it is necessary to consider the structure to better determine their stability. Our analysis indicates that only $(T \downarrow)_{6}\left(\mathrm{D} \downarrow_{3}\right)_{4}$ is stable. The same behavior, as far as the energy is concerned, is reproduced for $(\mathrm{T} \downarrow)_{N}\left(\mathrm{D} \downarrow_{3}\right)_{5}$ with $N \leq 5$ : as the separation of the $\mathrm{D} \downarrow$ atoms is growing along the simulation we are led to consider them unstable. We expect that all other clusters $(\mathrm{T} \downarrow)_{N}(\mathrm{D} \downarrow)_{M}$, with $N+M \leq 10$ and $M>5$ are unstable because the exchange of $\mathrm{T} \downarrow$ with the $\mathrm{D} \downarrow$ atoms does 
not change the interaction but raises the kinetic energy. The evolution of the energy of mixed clusters formed by $N$ tritium atoms and one hydrogen atom or up to four deuterium atoms is shown in Fig. 2 .

In addition to the energy, DMC simulations allow also for exact estimations of other relevant magnitudes such as the distribution of interparticle distances $P(r)$ or the distribution of particles with respect to the center of mass of the cluster $\rho(r)$. In both cases it is possible to eliminate the bias coming from the trial wave function by using pure estimators ${ }^{33}$ and arrive to exact results.

Our calculations confirm that the pure $\mathrm{T} \downarrow$ clusters are spatially very diffuse ${ }^{10}$ From the DMC results for $P(r)$ one can see that the average separation between particles $\left\langle r_{T T}\right\rangle$ ranges between $34 \AA$ in the case of $(\mathrm{T} \downarrow)_{3}$ to $10.9 \AA$ for $(\mathrm{T} \downarrow)_{6}$ and $10.5 \AA$ for $(\mathrm{T} \downarrow)_{10}$. The addition of one $\mathrm{D} \downarrow$ or one $\mathrm{H} \downarrow$ reduces the average separation between $\mathrm{T} \downarrow$ atoms in the case of clusters with $N=3$ or 4 , as can be seen in Fig. [3, which compares the pair distribution functions of $(T \downarrow)_{3},(T \downarrow)_{3} \mathrm{D} \downarrow$ and $(T \downarrow)_{3} H \downarrow$. In contrast, for clusters with more $T \downarrow$ atoms the $T \downarrow-T \downarrow$ separation remains almost unaffected by the addition of one $\mathrm{D} \downarrow$ or one $\mathrm{H} \downarrow$ atom. Due to the larger zero-point motion of $\mathrm{D} \downarrow$ and $\mathrm{H} \downarrow$ atoms, $\left\langle r_{T T}\right\rangle>\left\langle r_{T D}\right\rangle>\left\langle r_{T H}\right\rangle$. Moreover, we obtain $\left\langle r_{T H}\right\rangle$ greater than $100 \AA$ indicating that the cluster $(\mathrm{T} \downarrow)_{3} \mathrm{H} \downarrow$ is unstable. Similar behavior is noticed for larger $(\mathrm{T} \downarrow)_{N} \mathrm{H} \downarrow$ clusters.

The effect of adding $\mathrm{D} \downarrow$ atoms to the core of $(\mathrm{T} \downarrow)_{6}$ cluster is shown in Fig. 4, The separation between pairs of $\mathrm{T} \downarrow$ atoms remains almost unchanged, while the $\mathrm{T} \downarrow-\mathrm{D} \downarrow$ and $\mathrm{D} \downarrow$ $\mathrm{D} \downarrow$ separations grow with the addition of $\mathrm{D} \downarrow$ atoms. There is a noticeable difference between clusters with $\mathrm{D} \downarrow_{2}$ and $\mathrm{D} \downarrow_{3}$ spin-polarized deuterium. Former clusters with more than three particles are much more extended, i.e., the average separation between $\mathrm{D} \downarrow$ atoms is larger and the tail of the distribution decays slowly. Despite the large size of the cluster, the average separation between particles does not appear to grow in the case of $(\mathrm{T} \downarrow)_{6}\left(\mathrm{D} \downarrow_{2}\right)_{3}$ so we are led to consider this cluster as stable; the same conclusion applies to $(\mathrm{T} \downarrow)_{6}\left(\mathrm{D} \downarrow_{3}\right)_{4}$. On the other hand, the average $\mathrm{D} \downarrow$ - $\mathrm{D} \downarrow$ distance in the $(\mathrm{T} \downarrow)_{6}\left(\mathrm{D} \downarrow_{2}\right)_{4}$ cluster is slightly above $40 \AA$ but grows very slowly in the course of the simulation, indicating that it could be unstable.

Finally, Fig. 5 presents the density distribution of the clusters $(\mathrm{T} \downarrow)_{N}(\mathrm{D} \downarrow)$, for $N=3,6,9$. With the increase in the number of bosons, deuterium is pushed to the surface of the cluster, although even for $N=9$ there is still an appreciable probability of finding it inside the cluster. Similar behavior is found for $(\mathrm{T} \downarrow)_{N}(\mathrm{H} \downarrow)$ clusters. For larger clusters, one can expect that both $\mathrm{D} \downarrow$ and $\mathrm{H} \downarrow$ will be pushed to the surface forming the so-called Andreev states,,$^{34}$ similarly to the well known behavior of ${ }^{3} \mathrm{He}$ in mixed ${ }^{3} \mathrm{He}-{ }^{4} \mathrm{He}$ clusters. $\underline{15}^{15}$

\section{CONCLUSIONS}

The ground-state properties of spin-polarized pure and mixed tritium clusters have been accurately determined using the DMC method. Our results show that the trimer is a Borromean or halo state in agreement with the results of Ref. 10. The most promising candidates for the super-Borromean states in mixed clusters have been $(T \downarrow)_{2}(\mathrm{D} \downarrow)_{1,2,3,4,5}$, but after a careful analysis we have found no bound states in either of these clusters. On the other hand, we conclude that it takes at least three $\mathrm{T} \downarrow$ atoms in order to bind 1-3 $\mathrm{D} \downarrow$ atoms, the case of $(T \downarrow)_{3}(\mathrm{D} \downarrow)_{3}$ being bound only when the deuterium atoms are of $\mathrm{D} \downarrow_{3}$ type. The $\mathrm{DMC}$ results show that $6 \mathrm{~T} \downarrow$ atoms are needed in order to bind $4 \mathrm{D} \downarrow_{3}$ atoms, while clusters

with up to total 10 atoms and more than $4 \mathrm{D} \downarrow$ atoms seem all to be unstable. We have not considered mixed clusters with the $\mathrm{D} \downarrow$ atoms in only one nuclear spin-state $\mathrm{D} \downarrow_{1}$ since, from 
analogy with the bulk, we expect them to be less bound than $\mathrm{D} \downarrow_{2}$ and $\mathrm{D} \downarrow_{3}$.

Finally, and concerning mixed tritium-hydrogen clusters, our results show that all the $(\mathrm{T} \downarrow)_{N}(\mathrm{H} \downarrow)_{M}$ clusters with $N+M \leq 11$ are unstable. Work is in progress to find out how many $\mathrm{T} \downarrow$ atoms are needed to bind one $\mathrm{H} \downarrow$ atom.

\section{Acknowledgments}

J. B. acknowledges support from DGI (Spain) Grant No. FIS2005-04181 and Generalitat de Catalunya Grant No. 2005SGR-00779. I.B and L.V.M. acknowledge support from MSES (Croatia) Grant No. 177-1770508-0493. We also acknowledge the support of the Central Computing Services at the Johannes Kepler University in Linz, where part of the computations was performed.

1 L. Vranješ Markić, J. Boronat and J. Casulleras, Phys. Rev. B 75, 064506 (2007).

2 W. C. Stwaley and L. H. Nosanow, Phys. Rev. Lett. 36, 910 (1976).

3 D. G. Fried, T. C. Killian, L. Wilmann, D. Landhuis, S. C. Moss, P. Kleppner, and T. J. Greytak, Phys. Rev. Lett. 81, 3811 (1998).

4 R. M. Panoff and J. W. Clark, Phys. Rev. B 365527 (1987).

5 M. F. Flynn, J. W. Clark, E. Krotscheck, R. A. Smith, and R. M. Panoff, Phys. Rev. B 32, 2945 (1985).

6 B. Skjetne and E. Østgaard, J. Phys.: Condens. Matter 118017 (1999).

7 R. D. Etters, J. V. Dugan, Jr., and R. W. Palmer, J. Chem. Phys. 62, 313 (1975).

8 M. D. Miller, L. H. Nosanow, Phys. Rev. B 15, 4376 (1976).

9 B. R. Joudeh, M. K. Al-Sugheir, H. B. Ghassib, Physica B 388, 237 (2007).

10 D. Blume, B. D. Esry, Chris H. Greene, N. N. Klausen, and G. J. Hanna, Phys. Rev. Lett. 89, $163402(2002)$.

11 D. V. Fedorov, A. S. Jensen and K. Riisager, Phys. Rev. C 49, 201 (1994); 50, 2372 (1994).

12 A. S. Jensen, K. Riisager, D. V. Fedorov and E. Garrido, Rev. Mod. Phys. 76, 215 (2004).

13 M. Salci, Sergey B. Levin and Nils Elander, Phys. Rev. A 69, 044501(2004).

14 A. Kalinin, O. Kornilov, W. Schölkopf and J. P. Toennies, Phys. Rev. Lett. 95, 113402 (2005).

15 M. Barranco, R. Guardiola, S. Hernández, R. Mayol, and M. Pi, J. Low Temp. Phys. 142,1 (2006), and references therein.

16 S. Fantoni, R. Guardiola, J. Navarro, A. Zuker, J. Chem. Phys. 123, 054503 (2005).

17 I. Bešlić, L. Vranješ Markić, S. Kilić, J. Low Temp. Phys. 143, 257 (2006).

18 E. Sola, J. Casulleras and J. Boronat, Phys. Rev. B 73, 092515 (2006).

19 G. J. Hanna and D. Blume, Phys. Rev. A 74, 063604 (2006).

20 See, e.g., S. Giorgini, L. P. Pitaevskii, and S. Stringari, preprint, cond-mat/0706.3360.

21 V. Efimov, Phys. Lett. 33B, 563 (1970).

22 T. Kraemer, M. Mark, P. Waldburger, J. G. Danzl, C. Chin, B. Engeser, A. D. Lange, K. Pilch, A. Jaakkola, H.-C. Nägerl, and R. Grimm, Nature (London) 440, 315 (2006).

23 Yong Li, Qungdong Gou and Tingyun Shi, Phys. Rev. A 74, 032502 (2006).

24 W. Kolos and L. Wolniewicz, J. Chem. Phys. 43, 2429 (1965); Chem. Phys. Lett. 24, 457 (1974).

25 M. J. Jamieson, A. Dalgarno, and L. Wolniewicz, Phys. Rev. A 61, 042705 (2000). 
26 Zong-Chao Yan, James F. Babb, A. Dalgarno, and G. W. F. Drake, Phys. Rev A 54, 2824(1996).

27 W. Kolos and J. Rychlewski, J. Mol. Spectrosc. 143, 237 (1990).

28 J. Boronat and J. Casulleras, Phys. Rev. B 498920 (1994).

29 P. J. Reynolds, D. M. Ceperley, B. J. Alder, and W. A. Lester Jr., J. Chem. Phys. 77, 5593 (1982).

30 J. Boronat and J. Casulleras, Europhys. Lett. 38, 291 (1997).

31 T. I. Sachse, K.T. Tang, and J. P. Toennies, Chem. Phys. Lett. 317, 346 (2000).

32 I. F. Silvera and V.V. Goldman, Phys. Rev. Lett. 45915 (1980).

33 J. Casulleras and J. Boronat, Phys. Rev. B 52, 3654 (1995).

34 A. F. Andreev, Sov. Phys. JETP 23, 939 (1966).

Figure captions

FIG. 1: Comparison of our results for spin-polarized tritium clusters (circles) with the results of Blume et. al. $\underline{\underline{10}}$ (crosses). The difference between our results and the ones from Blume et. $\quad a l . \frac{10}{n}$ is mainly due to the use of a slightly different interaction (see text). The error bars of the DMC energies are smaller than the size of the symbols.

FIG. 2: Energies of the spin-polarized pure and mixed tritium clusters in $\mathrm{K}$ as a function of the number of $\mathrm{T} \downarrow$ atoms.

FIG. 3: Distribution of interparticle distances in the $(\mathrm{T} \downarrow)_{3},(\mathrm{~T} \downarrow)_{3} \mathrm{D} \downarrow$ and $(\mathrm{T} \downarrow)_{3} \mathrm{H} \downarrow$ clusters.

FIG. 4: Distribution of interparticle distances of the $(\mathrm{T} \downarrow)_{6}(\mathrm{D} \downarrow)_{N}$ clusters. Full line denotes $\mathrm{T} \downarrow-\mathrm{T} \downarrow$, dashed line $\mathrm{T} \downarrow-\mathrm{D} \downarrow$, and dotted line $\mathrm{D} \downarrow-\mathrm{D} \downarrow$ separation. $N=3,4$ distributions with lower maxima in $\mathrm{D} \downarrow-\mathrm{D} \downarrow$ separations correspond to $\mathrm{D} \downarrow_{2}$ and those with higher maxima to $\mathrm{D} \downarrow_{3}$.

FIG. 5: Density distributions of $\mathrm{T} \downarrow$ (upper curves) and $\mathrm{D} \downarrow$ (lower curves) for $(\mathrm{T} \downarrow)_{N} \mathrm{D} \downarrow$ clusters. 


\begin{tabular}{c|cc}
$\mathrm{N}$ & $(\mathrm{T} \downarrow)_{N}$ & $(\mathrm{~T} \downarrow)_{N} \mathrm{H} \downarrow$ \\
\hline 2 & - & - \\
3 & $-4.8(0.2)$ & $-4.7(0.7)$ \\
4 & $-126(2)$ & $-126(1)$ \\
5 & $-398(1)$ & $-398(2)$ \\
6 & $-810(2)$ & $-807(3)$ \\
7 & $-1348(4)$ & $-1339(6)$ \\
8 & $-1991(5)$ & $-1982(7)$ \\
9 & $-2727(7)$ & $-2720(9)$ \\
10 & $-3553(8)$ &
\end{tabular}

TABLE I: The ground state energy (in $\mathrm{mK}$ ) of pure spin-polarized tritium clusters with $N$ atoms and tritium clusters with an additional hydrogen atom. Clusters which appear to be unstable are written in italic. Figures in parenthesis are the statistical errors.

\begin{tabular}{|c|c|c|c|c|c|c|c|}
\hline \multicolumn{8}{|c|}{$\mathrm{E}\left[(\mathrm{T} \downarrow)_{N}(\mathrm{D} \downarrow)_{M}\right]$} \\
\hline $\mathrm{N}$ & $\mathrm{D} \downarrow$ & $(\mathrm{D} \downarrow)_{2}$ & $\left(\mathrm{D} \downarrow_{2}\right)_{3}$ & $\left(\mathrm{D} \downarrow_{2}\right)_{4}$ & $\left(\mathrm{D} \downarrow_{3}\right)_{3}$ & $\left(\mathrm{D} \downarrow_{3}\right)_{4}$ & $\overline{\left(\mathrm{D} \downarrow_{3}\right)_{5}}$ \\
\hline 2 & - & - & - & - & - & - & - \\
\hline 3 & $-12.2(0.9)$ & $-31(3)$ & $-27(3)$ & $-21(2)$ & $-56(2)$ & $-58(5)$ & $-46(6)$ \\
\hline 4 & $-182(3)$ & $-256(3)$ & $-253(4)$ & $-241(3)$ & $-336(3)$ & $-327(6)$ & $-313(6)$ \\
\hline 5 & $-510(4)$ & $-629(4)$ & $-633(6)$ & $-610(6)$ & $-769(4)$ & $-755(7)$ & $-732(6)$ \\
\hline 6 & $-972(4)$ & $-1139(3)$ & $-1145(9)$ & $-1135(5)$ & $-1322(5)$ & $-1326(10)$ & \\
\hline 7 & $-1552(4)$ & $-1763(10)$ & $-1747(15)$ & & $-1989(7)$ & & \\
\hline 8 & $-2237(6)$ & $-2492(12)$ & $-2554(15)$ & & $-2755(8)$ & & \\
\hline 9 & $-3013(7)$ & & & & & & \\
\hline
\end{tabular}

TABLE II: The ground-state energy $E$ (in $\mathrm{mK}$ ) of mixed spin-polarized tritium-deuterium clusters. For mixed clusters having more than two deuterium atoms, combinations with $\mathrm{D}_{\downarrow_{2}}$ and $\mathrm{D} \downarrow_{3}$ are considered. The clusters who are at the threshold of binding or appear to be unstable are written in italic. Figures in parenthesis are the statistical errors.

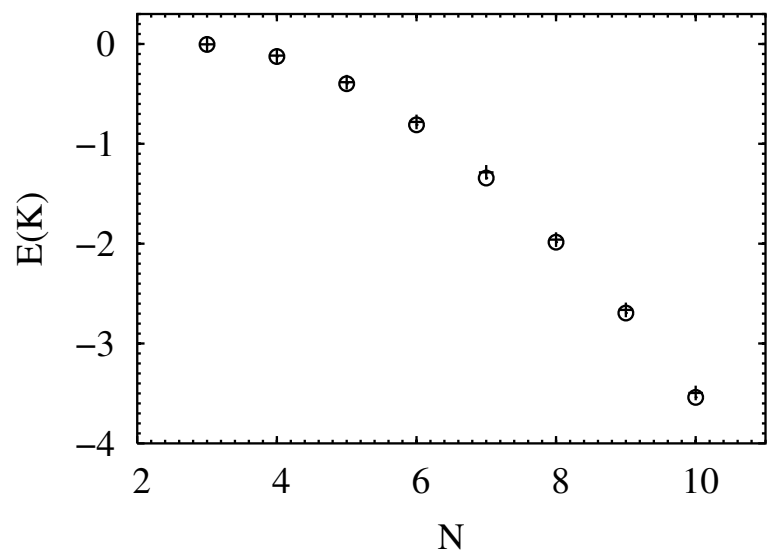

FIG. 1: I. Bešlić et al. 


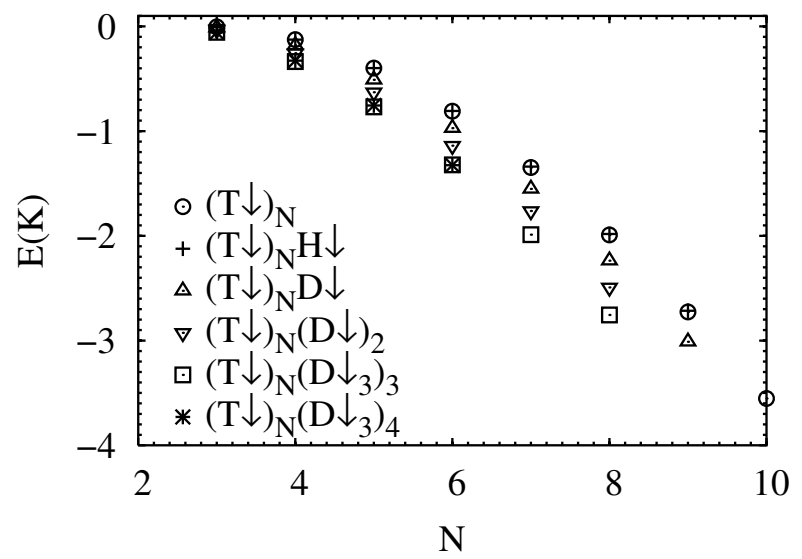

FIG. 2: I. Bešlić et al.

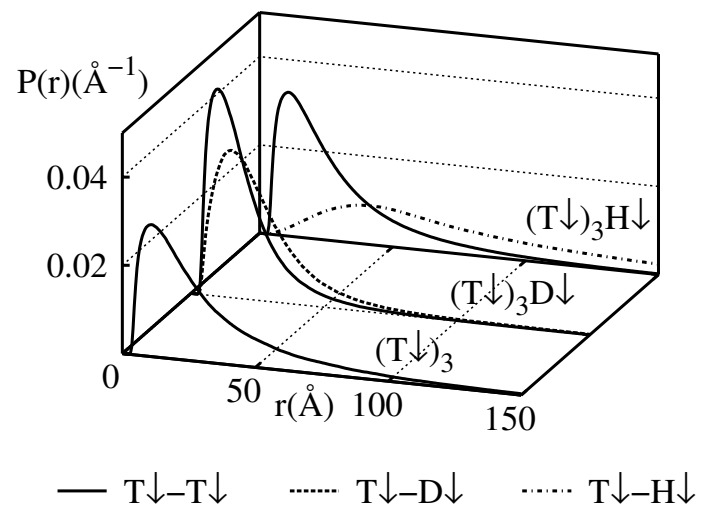

FIG. 3: I. Bešlić et al.

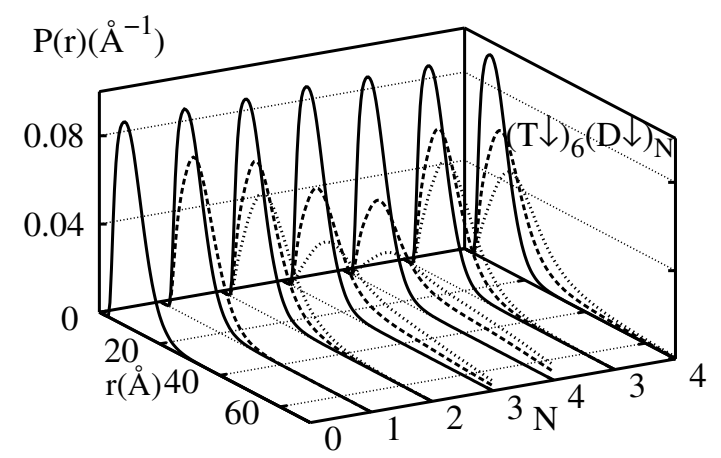

FIG. 4: I. Bešlić et al. 


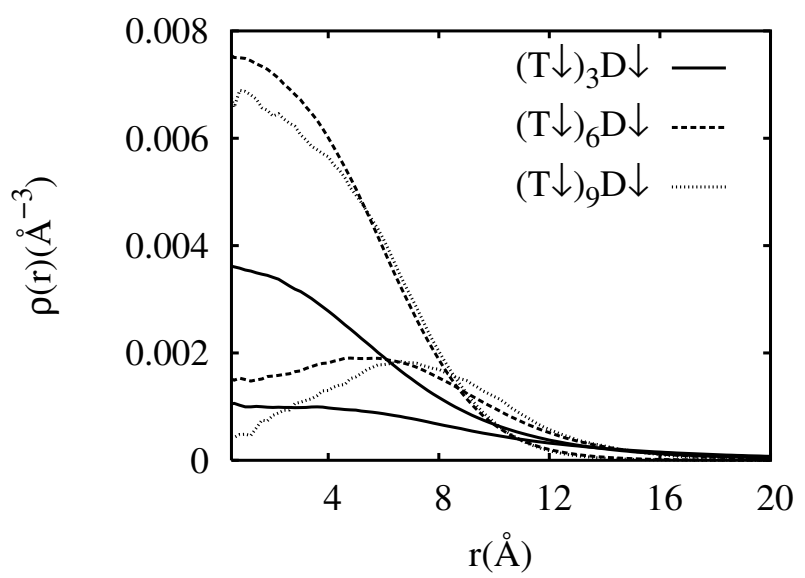

FIG. 5: I. Bešlić et al. 